\title{
DESEMPENHO AGRONÔMICO DE CAPIM NAPIER (Pennisetum purpureum) CULTIVADO EM SISTEMAS ALAGADOS CONSTRUÍDOS
}

\author{
Antonio Teixeira de Matos $^{1}$, Sérgio Silva Abrahão², Odilon Gomes Pereira ${ }^{3}$
}

\section{RESUMO}

O objetivo, com a realização deste trabalho, foi estudar o desempenho de Sistemas Alagados Construídos (SACs), cultivados com capim elefante cv. Napier (Pennisetum purpureum), submetidos a diferentes taxas de carregamento orgânico (TCOs), proporcionadas pela aplicação de águas residuárias da indústria de laticínios (ARL), nas condições climáticas de Viçosa - MG. A infraestrutura experimental foi constituída por cinco SACs com fluxo subsuperficial horizontal, constituídos por tanques de 0,40 × 0,75 × 3,00 m, impermeabilizados e preenchidos com brita zero e profundidade do leito filtrante de $0,33 \mathrm{~m}$. A ARL foi aplicada nos SACs de forma a promover seu escoamento de forma subsuperficial, com vazão média de $60 \mathrm{~L} \cdot \mathrm{d}^{-1}$ e tempo de detenção hidráulica igual a 4,8 dias. As TCOs aplicadas foram de 66, 130, 190, 320 e $570 \mathrm{~kg} \cdot \mathrm{ha}^{-1} \cdot \mathrm{d}^{-1}$ de DBO. O capim Napier se adaptou bem aos SACs, apresentando bom enraizamento, embora predominantemente superficial, grande produtividade e capacidade de remover nutrientes, especialmente nitrogênio e potássio da ARL.

Palavras-chave: wetlands construídos, águas residuárias, leitos cultivados, processamento do leite.

\section{ABSTRACT \\ AGRONOMIC PERFORMANCE OF PENNISETUM PURPUREUM CULTIVATED IN CONSTRUCTED WETLANDS}

The study was carried out to evaluate the performance of the Pennisetum purpureum cultivated in constructed wetlands (CWs) subjected to different organic loading rate (OLS), prepared by mixing milk-processing wastewater and water (MPW), under the climatic conditions of Viçosa-MG. The experimental structure consisted of five CWs with horizontal sub-surface flow. Waterproof tanks $(0.40 \times 0.75 \times 3.00 \mathrm{~m})$ were filled with fine stones to the depth of $0.33 \mathrm{~m}$. The MPW was applied at the mean flow rate of $60 \mathrm{~L}^{-d^{-1}}$, with water retention time of 4.8 days and OLR of 66, 130, 190, 320 or $570 \mathrm{~kg} \cdot \mathrm{ha}^{-1}$.day ${ }^{-1}$ of BOD. Good rooting, although predominantly superficial, high yield and nutrient removal capacity, especially nitrogen and potassium, from MPW indicates good adaptation of P. purpureum to CWs.

Keywords: wastewater, constructed wetlands, milk processing.

\section{Recebido para publicação em 16/07/2008. Aprovado em 19/04/2011.}

1- Engenheiro Agrícola, D.Sc., Professor do Departamento de Engenharia Agrícola, Universidade Federal de Viçosa, atmatos@ufv.br

2- Engenheiro Agrícola, M.Sc. em Engenharia Agrícola, Departamento de Engenharia Agrícola, UFV

3- Agrônomo, D.Sc., Professor do Departamento de Zootecnia, Universidade Federal de Viçosa, UFV 


\section{INTRODUÇÃOO}

As águas residuárias oriundas das indústrias de laticínios (ARL) são constituídas por leite e seus subprodutos, além de detergentes, desinfetantes, areia, lubrificantes, açúcar, pedaços de frutas (em caso da produção de iogurte), essências e condimentos diversos (no caso da produção de queijos e manteiga), que são diluídos nas águas de lavagem de equipamentos, tubulações, pisos e demais instalações da indústria (MACHADO et al., 2002). O volume gerado de ARL pode variar de 1 a 5 vezes o volume de leite processado, dependendo do produto final produzido e do nível tecnológico da indústria de laticínios.

Entre as alternativas de baixo custo passíveis de se utilizar para o tratamento da ARL, estão os Sistemas Alagados Construídos (SACs) ou sistemas Wetland. Os SACs são sistemas projetados para utilizar plantas cultivadas em substratos (solo, areia, brita ou cascalho), onde, naturalmente e sob condições ambientais adequadas, ocorrem processos físicos, químicos e bioquímicos de tratamento das águas residuárias. Entretanto, existem poucos trabalhos para identificação das plantas mais recomendadas para uso nesses sistemas de tratamento.

Há, entre pesquisadores e projetistas, preferência na utilização de macrófitas em SACs, por serem plantas naturalmente adaptadas para o crescimento na água e em solos saturados. Entre as macrófitas utilizadas, tem sido comum o cultivo de espécies de maior porte, que são as plantas aquáticas vasculares florescentes, tal como a Typha sp. (HUSSAR, 2001; BRASIL, 2005), porém pouco tem sido estudado com referência à produção de espécies de valor comercial ou que possam ser usadas para alimentação animal.

Segundo Paganini (1997), as espécies vegetais a serem selecionadas devem ser perenes, ter alta tolerância ao excesso de água e a ambientes eutrofizados, ser de fácil propagação e crescimento rápido, ser de fácil colheita e manejo e possuírem alta capacidade de remoção de nutrientes e poluentes. Além disso, devem apresentar denso sistema radicular, ser pouco susceptível a pragas e doenças e ser passível de cortes sucessivos e frequentes.
O gênero Pennisetum é constituído por mais de 140 espécies (BRUNKEN, 1977), que se encontram amplamente distribuídas por todas as regiões tropicais do planeta. Dentro da espécie, é encontrada grande diversidade de formas, tipos ou variedades, apresentando grande variabilidade genética para a maior parte dos caracteres de interesse. $\mathrm{O}$ grupo de cultivares Napier apresenta variedades com touceiras abertas, colmos grossos, folhas largas e época de florescimento intermediária (abril a maio). Variedades do grupo Napier têm sido recomendadas, tanto para capineiras (corte) como para formação de pastagens.

O capim elefante é planta exigente em nutrientes, em decorrência de seu elevado potencial de produção. Como regra geral, a extração de nutrientes pela forrageira é proporcional aos rendimentos obtidos.

Aguiar et al. (1999) avaliaram a produção e o desempenho do capim elefante (Pennisetum purpureum, Schum.) fertirrigado com efluente de reatores anaeróbios obtendo relativamente grande produção de matéria seca, proteína bruta, altura das plantas e número de perfilhos por $\mathrm{m}^{2}$.

No presente trabalho, teve-se como objetivo estudar o desempenho agronômico do capimelefante cv. Napier (Pennisetum purpureum), quando cultivado em Sistemas Alagados Construídos submetidos a diferentes taxas de carregamento orgânico, proporcionadas pela aplicação de águas residuárias da indústria de laticínios, nas condições climáticas de Viçosa MG.

\section{MATERIAL E MÉTODOS}

O experimento foi conduzido na Área Experimental de Hidráulica, Irrigação e Drenagem do Departamento de Engenharia Agrícola da UFV, em Viçosa - MG. Para a realização do estudo, foi utilizada água residuária bruta proveniente da Usina de Beneficiamento de Leite da Fundação Arthur Bernardes (Laticínios da FUNARBE), localizada no Campus da UFV.

A infraestrutura experimental foi constituída por cinco leitos de sistemas alagados construídos (SACs) com fluxo subsuperficial horizontal. Cada SAC foi constituído por um tanque de $0,40 \times 0,75$ 
x 3,00 m, impermeabilizado, preenchido com substrato de brita zero, declividade de base de $0,5 \%$ no sentido longitudinal do tanque e profundidade do leito filtrante de $0,33 \mathrm{~m}$.

Para facilitar o pegamento das mudas de capim, os SACs foram previamente saturados com água residuária gerada na suinocultura da UFV, posteriormente foi feito o plantio, utilizando-se propágulos vegetativos (rizomas+caule) com, aproximadamente, $0,30 \mathrm{~m}$ de comprimento. A densidade de plantio foi, em média, 14 propágulos. $\mathrm{m}^{-2}$, e o plantio ocorreu em apenas uma etapa, no dia 16 de abril de 2005.

Após o plantio dos propágulos, deu-se continuidade ao fornecimento da água residuária proveniente da suinocultura nos SACs com e sem vegetação, com uma frequência de 3 vezes por semana, até o dia 03 de agosto de 2005. A partir do dia 04 de agosto de 2005, passou-se a aplicar, na mesma frequência, apenas água "limpa" do manancial (AM), e no dia 19 de agosto de 2005, iniciou-se a operação nos SACs, com aplicação diária da água residuária do laticínios (ARL).

A água residuária afluente aos SACs foi aplicada de forma subsuperficial, com vazão média de 60 L.d ${ }^{-1}$ e tempo de detenção hidráulica de 4,8 dias nos tanques. As taxas de carregamento orgânico (TCOs) aplicadas em cada tratamento foram de $66,130,190,320$ e $570 \mathrm{~kg} \cdot \mathrm{ha}^{-1} \cdot \mathrm{d}^{-1}$ de $\mathrm{DBO}$, denominados respectivamente, de CE-66, CE-130, CE-190, CE-320 e CE-570. As diferentes TCO dos afluentes foram obtidas por meio de misturas da água residuária de laticínio (ARL) bruta, que tinha DBO de aproximadamente 2.500 mg. $\mathrm{L}^{-1}$, com água "limpa" do manancial (AM).

As principais características físicas, químicas e bioquímicas da água residuária do laticínios (ARL) e da água "limpa" de manancial (AM), utilizadas para a dosagem das TCOs, estão apresentadas no Quadro 1.

A coleta da biomassa foi realizada 61 dias após ter sido realizado um corte de uniformização dos estandes de plantas, sendo constituída pelo corte das plantas de toda área de cada SAC, que foi subdividida em três subáreas (início, meio e fim), numa altura entre 5 e $10 \mathrm{~cm}$ acima do meio suporte. A quantificação da concentração de N-Total, P-Total, Potássio e Sódio no tecido vegetal do capim Napier foi feita em conformidade com as recomendações de Kiehl (1985), no Laboratório de Química dos

Quadro 1. Características físicas, químicas e bioquímicas da água residuária do laticínio (ARL) e da água "limpa" de manancial (AM)

\begin{tabular}{ccc}
\hline \multirow{2}{*}{ Variáveis } & ARL & AM \\
\cline { 2 - 3 } pH & \multicolumn{2}{c}{ Média e Desvio Padrão } \\
\hline CE $\left(\mu \mathrm{S} . \mathrm{cm}^{-1}\right)$ & $3,78 \pm 0,47$ & $6,94 \pm 0,17$ \\
DBO $\left(\mathrm{mg} \cdot \mathrm{L}^{-1}\right)^{*}$ & $2790 \pm 633$ & $89 \pm 14$ \\
DQO $\left(\mathrm{mg} \cdot \mathrm{L}^{-1}\right)$ & $5143 \pm 328$ & $12 \pm 4$ \\
SS $\left(\mathrm{mg} \cdot \mathrm{L}^{-1}\right)$ & $702 \pm 100$ & $20 \pm 6$ \\
ST $\left(\mathrm{mg} \cdot \mathrm{L}^{-1}\right)$ & $2407 \pm 442$ & $3 \pm 3$ \\
SD $\left(\mathrm{mg} \cdot \mathrm{L}^{-1}\right)$ & $1705 \pm 479$ & $104 \pm 41$ \\
N-total $\left(\mathrm{mg} \cdot \mathrm{L}^{-1}\right)$ & $89,54 \pm 17,3$ & $101 \pm 43$ \\
P-total $\left(\mathrm{mg} \cdot \mathrm{L}^{-1}\right)$ & $22,51 \pm 5,1$ & $3,33 \pm 5,7$ \\
Potássio $\left(\mathrm{mg} \cdot \mathrm{L}^{-1}\right)$ & $66,36 \pm 23,9$ & $0,12 \pm 0,17$ \\
Sódio $\left(\mathrm{mg} \cdot \mathrm{L}^{-1}\right)$ & $107,72 \pm 98,4$ & $3,77 \pm 1,5$ \\
& & $8,43 \pm 4,2$
\end{tabular}

*Variável utilizada para a dosagem das TCOs 
Resíduos do Departamento de Engenharia Agrícola da UFV. O conteúdo de proteína bruta foi obtido com a multiplicação da concentração de N-total por 6,25, conforme recomendado por Silva (1998).

No final do período de avaliação dos SACs, foram feitas medidas da profundidade atingida pelas raízes da plantas dentro dos SACs, com a remoção cuidadosa da brita do entorno do sistema radicular das plantas, liberando-o.

Os resultados foram interpretados quantitativamente, utilizando-se, para isso, o ajuste de equações matemáticas, escolhidas com base no valor do coeficiente de determinação $\left(R^{2}\right)$, desde que houvesse lógica para o tipo de comportamento descrito.

\section{RESULTADOS E DISCUSSÃO}

No período anterior à partida do sistema com a ARL, não foram observados sintomas de deficiência nutricional nem distúrbios de insanidade no estande das plantas. Porém, três dias após o início da aplicação da ARL, observouse amarelecimento das folhas do capim Napier cultivado nos SACs operados com TCOs de 190, 320, e 570.kg.ha-1. $\mathrm{d}^{-1}$ de DBO. Entretanto, ao final da terceira semana do início da partida do sistema, estas plantas já apresentavam aspecto normal, demonstrando capacidade de adaptação às novas condições de cultivo. Acredita-se que as mais altas condutividades elétricas nas soluções preparadas a partir da ARL bruta, além das relativamente altas concentrações de potássio e, principalmente, sódio, tenham proporcionado, inicialmente, problemas osmóticos às plantas. Com o passar do tempo, ao que tudo indica, mecanismos de restrição à entrada desses íons e, principalmente, associados à obtenção de novo equilíbrio osmótico da planta com a solução aplicada tenham sido fatores que proporcionaram adaptação das plantas às condições do meio.

Com exceção do SAC que recebeu maior TCO (CE-570), todos os outros SACs cultivados com capim Napier (CE-66, CE-130, CE-190, e CE-320) apresentaram aumento gradual na altura média das plantas à medida que se aproximava da zona de saída. No entanto, mesmo no SAC CE-570, verificou-se aumento da altura média das plantas no meio e na zona de saída quando comparada às obtidas na zona de entrada deste SAC (Figura 1a). A tendência de aumento na altura média das plantas na zona intermediária e de saída pode ser justificada pela degradação do material orgânico aportado ao sistema, o que possibilitou a disponibilização de nutrientes com a passagem da ARL através do meio suporte, com isso, maior crescimento das plantas. Na Figura $1 \mathrm{~b}$ está ilustrado o aspecto típico da zona radicular do

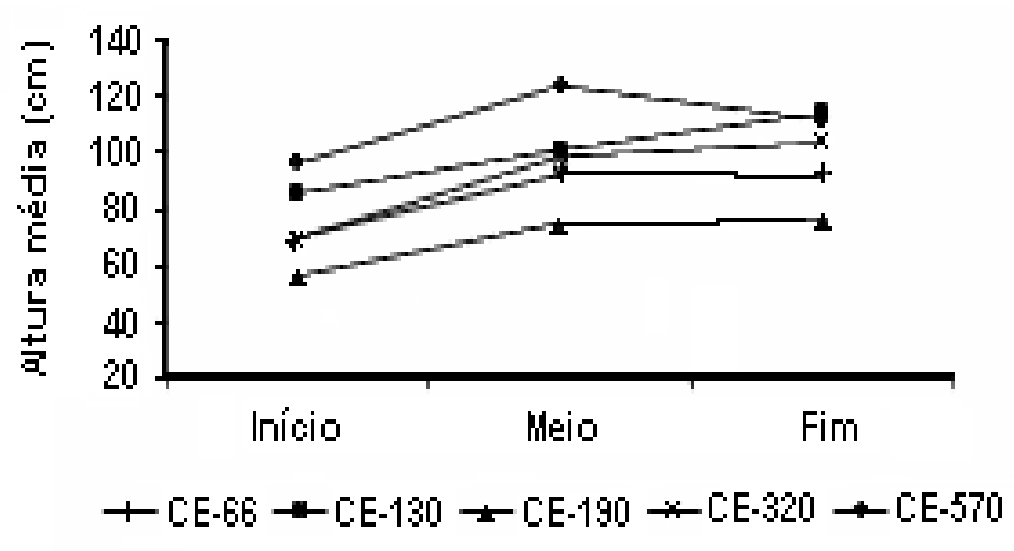

$\mathbf{a}$

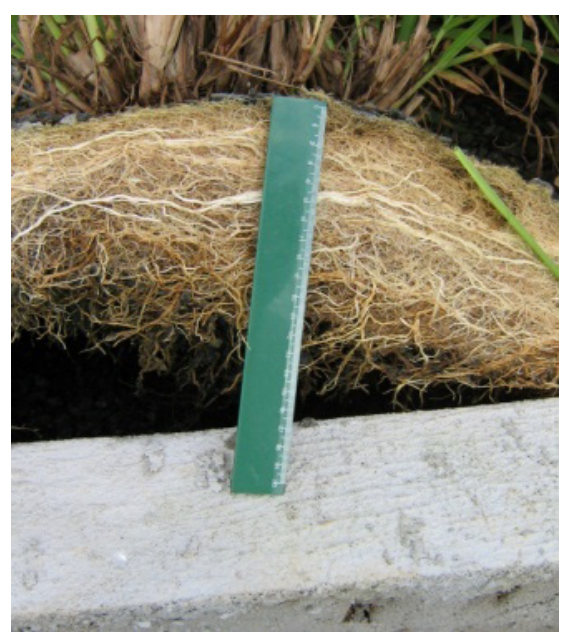

b

Figura 1. Altura média de plantas ao longo do comprimento dos SACs, aos 61 dias após o corte de homogeneização dos estandes (a), e aspecto típico do sistema radicular do capim Napier (b). 
capim Napier, desenvolvida durante o período de condução do experimento, podendo-se verificar a existência de densa trama de raízes superficiais no capim Napier.

Ao final do período de aplicação da ARL nos SACs, verficou-se que a profundidade máxima atingida pelo sistema radicular do capim Napier foi de $20 \mathrm{~cm}$, com forte concentração nos primeiros $3 \mathrm{~cm}$ da profundidade. Esse tipo de comportamento radicular pode ser um indicativo de que esse capim seria de cultivo recomendável em SACs de escoamento superficial, uma vez que dessa forma poderia proporcionar a remoção de maior quantidade de sólidos dissolvidos na água residuária.

$\mathrm{Na}$ Figura 2 apresenta-se a curva de produtividade acumulada de matéria seca em função das TCOs, obtida a partir das duas amostragens efetuadas em cada SAC cultivado com capim Napier. Os dados mostraram certa uniformidade na produtividade dos SACs CE-66, CE-130, CE320 e CE-570, mas decréscimo na produtividade no SAC CE-190. Como não houve ajuste de nenhuma equação matemática considerada modelo adequado para explicação do fenômeno biológico, foi apresentada a equação de uma reta indicando o valor médio de 7,65 t.ha ${ }^{-1}$ para qualquer TCO aplicada.

Acredita-se que a tendência de decréscimo na produtividade do capim com a aplicação das TCOs intermediárias possa estar relacionada com a disponibilidade de oxigênio dissolvido (OD) na água residuária em tratamento nos SACs. Supõese que os SACs que receberam menores taxas de carga orgânica (CE-66 e CE-130) tiveram maior disponibilidade de OD para a degradação da matéria orgânica aplicada e, consequentemente, maior disponibilidade de nutrientes para o capim Napier. No caso do SAC CE-190, que recebeu uma taxa de carga orgânica intermediária, especulase que o acúmulo de matéria orgânica tenha proporcionado ambiente extremamente anaeróbio, com pouca capacidade para disponibilizar nutrientes e, consequentemente, proporcionou baixa produtividade do capim Napier. Acredita-se que, apesar de os SACs CE-320 e CE-570 estarem sujeitos a taxas de carga orgânica superiores às recebidas pelo SAC CE-190, o maior aporte de nutrientes ao sistema pode ter contrabalançado os efeitos negativos da formação de um ambiente anaeróbio. Como dito anteriormente, formou-se uma trama de raízes muito densa na superfície dos SACs cultivados com capim Napier e isto proporcionou a retenção e degradação de matéria orgânica em ambiente mais aerado nos SACs CE-320 e CE-570, o que pode ter favorecido o desenvolvimento das plantas, a despeito do aumento da carga orgânica aplicada.

Como a produtividade média de matéria seca do capim Napier foi de 7,65 t.ha ${ }^{-1}$, se mantidos os mesmos intervalos entre cortes e produtividade do capim, isso representa 45,9 t.ha $^{-1} \cdot$ ano $^{-1}$. Vicente-Chandler et al. (1974), avaliando o capim elefante cultivado em solo que recebeu calagem e fertilizantes inorgânicos, obtiveram 28,2 t.ha ${ }^{-1}$ ano $^{-1}$ de matéria seca para capim colhido a cada 60 dias, valores que correspondem a cerca de $61 \%$ da produção obtida neste trabalho.

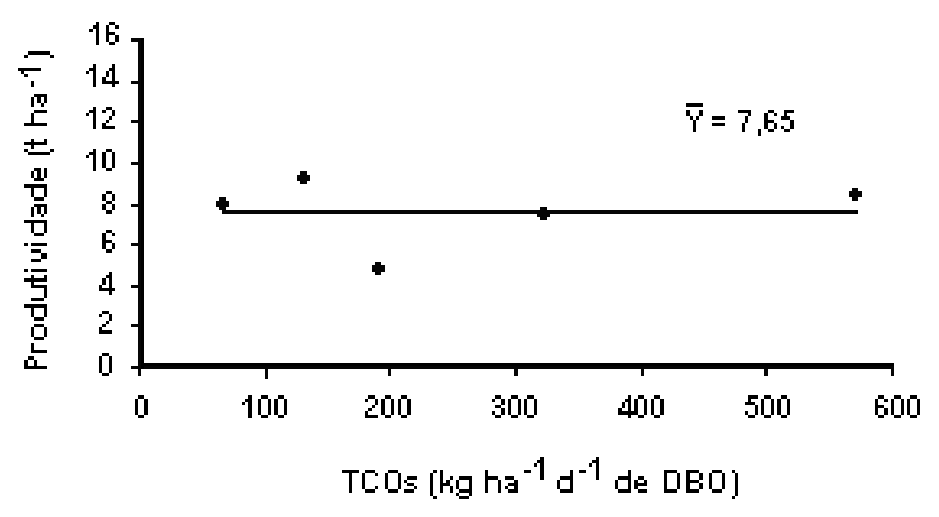

Figura 2. Produtividade de matéria seca do capim Napier em função das taxas de carga orgânica superficial (TCOs) aplicadas. 
Na Figura 3 estão apresentados os dados experimentais, as curvas e as equações ajustadas de concentração de $\mathrm{N}, \mathrm{P}$ e $\mathrm{Na}$ parte aérea do capim Napier, como função da TCO aplicada nos SACs. Verifica-se que houve tendência de aumento na concentração de N-total no tecido vegetal do capim Napier com o aumento nas TOCs aplicadas. O mesmo não se pode dizer para $\mathrm{P}$ e $\mathrm{Na}$, que apresentaram menores concentrações nas TCOs intermediárias.

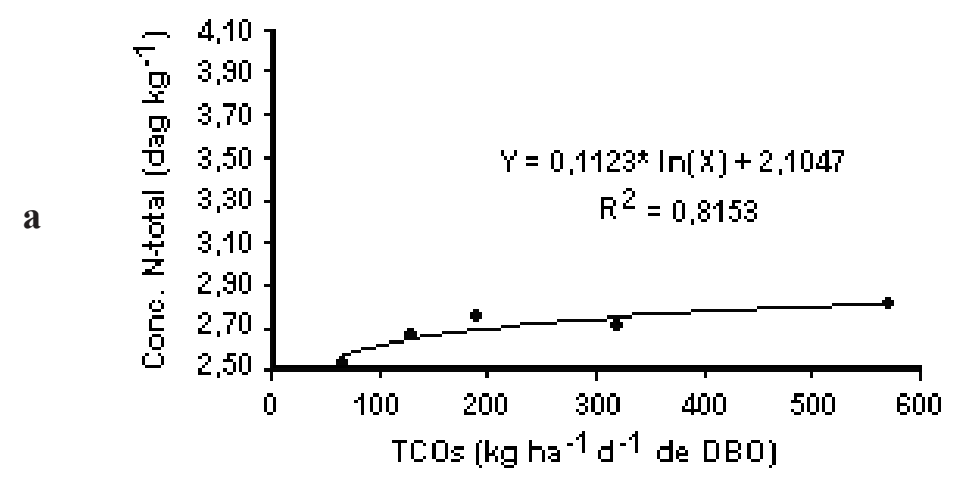

*: significativos em nível de $2 \%$ de probabilidade

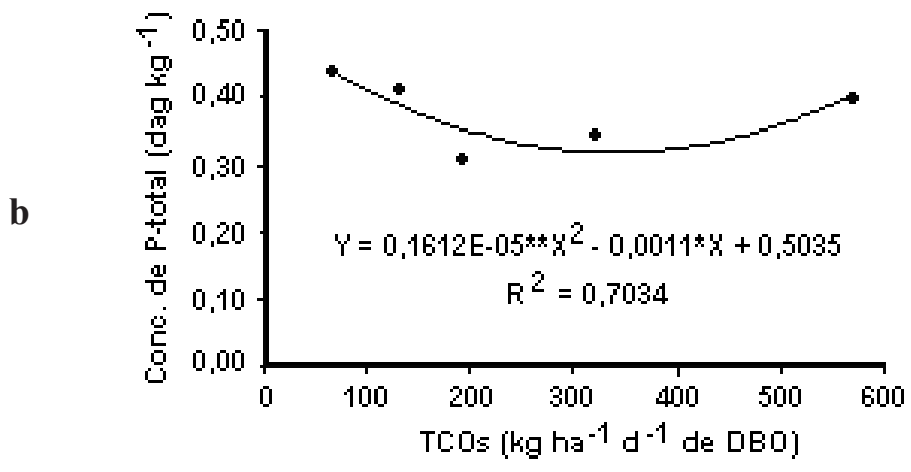

* e **: significativos em nível de $8 \%$ de probabilidade

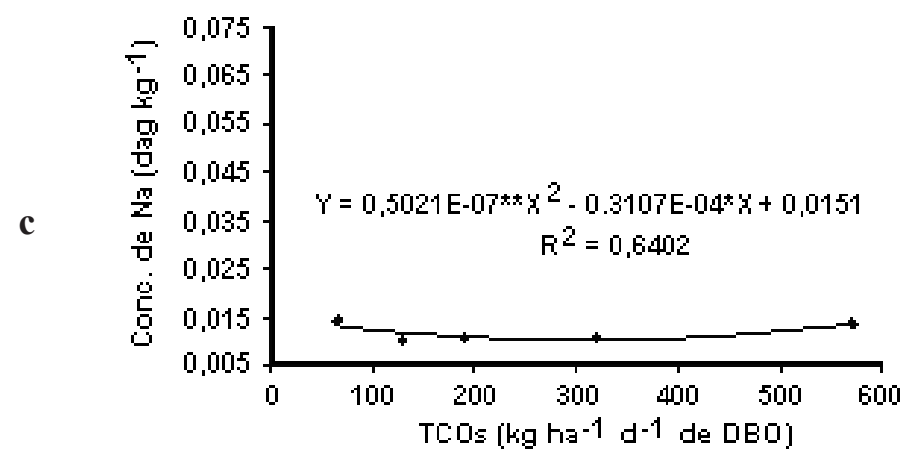

* e **: significativos em nível de $11 \%$ e $10 \%$ de probabilidade

Figura 3. Variação da concentração de N-total (a), $\mathrm{P}$ (b) e $\mathrm{Na}$ (c) na parte aérea do capim Napier em função das taxas de carga orgânica superficial (TCOs) aplicadas. 
A concentração de nitrogênio nas folhas do capim Napier aumentou até valores de taxa de carga orgânica próximos de $200 \mathrm{~kg} \cdot \mathrm{ha}^{-1} \cdot \mathrm{dia}^{-1} \mathrm{de}$ DBO, sendo que, a partir desta taxa, o aumento passou a ser mais moderado. A concentração de nitrogênio no capim Napier variou de 2,54 a 2,81 dag. $\mathrm{kg}^{-1}$, sendo estes valores correspondentes aos tratamentos CE-66 e CE-570, respectivamente. Calculando-se a concentração de proteína bruta no capim Napier, foram obtidos valores entre 15,9 a 17,6 dag. $\mathrm{kg}^{-1}$, considerados indicativos de capim com elevado valor nutricional para ruminantes.

A concentração de fósforo nas folhas do capim Napier diminuiu até valores próximos de $340 \mathrm{~kg} \cdot \mathrm{ha}^{-1} \cdot \mathrm{d}^{-1}$ de TCO e, a partir deste valor, observou-se tendência de aumento na concentração de P-total. Este comportamento é semelhante ao que ocorreu com a produção de matéria seca do capim Napier em função das TCOs aplicadas e pode ser explicado da mesma forma. Em vista disso, pode-se suspeitar que a deficiência na absorção de fósforo tenha sido importante fator para ter sido obtido o decréscimo na produtividade de matéria seca do capim Napier.

Supõe-se que a disponibilização de fósforo para absorção pelas plantas tenha sido influenciada, principalmente, pela quantidade de oxigênio dissolvido (OD) nos SACs, assim, em baixas TCOs houve menor consumo de OD para a degradação da matéria orgânica, consequentemente, proporcionando sua maior disponibilidade para a respiração radicular. Entretanto, a partir da TCO de $340 \mathrm{~kg} \cdot \mathrm{ha}^{-1} \cdot \mathrm{d}^{-1}$, a maior disponibilidade de fósforo no meio aparentemente compensou a deficiência de oxigênio na zona radicular das plantas.

Em relação à concentração de potássio, embora tenha variado de 1,38 a 2,32 dag. $\mathrm{kg}^{-1}$, não foi possível o ajuste de nenhuma equação matemática que explicasse o fenômeno, desta forma, considerou-se concentração constante e igual a 1,98 dag. $\mathrm{kg}^{-1}$. Houve, no entanto, tendência de redução nas concentrações de potássio à medida que foram aumentadas as TCOs aplicadas, até valores próximos de $300 \mathrm{~kg} \cdot \mathrm{ha}^{-1} \cdot \mathrm{d}^{-1} \mathrm{e}$, a partir destes valores, observou-se tendência de aumento na concentração deste nutriente. Considerandose as relativamente altas concentrações de sódio no afluente aos SACs e que o potássio sofre a concorrência desse cátion, prejudicando sua absorção pelas plantas, acredita-se que uma das razões para a observada tendência de decréscimo na absorção de potássio se deveu ao aumento na disponibilidade de sódio nos SACs, com o aumento das TCOs.

Gomide (1986) considerou uma concentração de potássio na faixa de 1,5 a 2,0 dag.kg-1 normal para forrageiras com bom suprimento de potássio e que valores mais elevados indicam absorção de luxo deste elemento pelas diferentes espécies forrageiras. Com base nisto, pode-se afirmar que as concentrações de potássio no capim Napier podem ser consideradas normais, à exceção das obtidas no material vegetal coletado no SAC que recebeu $190 \mathrm{~kg} \cdot \mathrm{ha}^{-1} \cdot \mathrm{d}^{-1}$ de DBO.

Houve pequena alteração na concentração de sódio nas folhas do capim Napier, tendo sido obtidos valores entre 0,010 e 0,015 dag. $\mathrm{kg}^{-1}$.

No Quadro 2 apresentam-se os valores de remoção de N-total, P-total, K e Na pelas plantas, em função das TCOs aplicadas em cada tratamento. Considerando que os valores apresentados no Quadro 2 são relativos a apenas 61 dias de cultivo do capim, pode-se considerar que os resultados obtidos sejam muito significativos, já que em um ano de cultivo nos SACs (mantendo-se o mesmo intervalo entre cortes), a remoção de nutrientes deverá ser de $1.236 \mathrm{~kg} \cdot \mathrm{ha}^{-1}$ de N; $180 \mathrm{~kg} \cdot \mathrm{ha}^{-1}$ de P; 894 kg.ha- ${ }^{-1}$ de K e 5,5 kg.ha-1 de Na. Gomes Filho (2000), avaliando sistema hidropônico de cultivo de capim congo (Brachiaria ruziziensis) para tratamento de água residuária da suinocultura (ARS), obteve remoções de $\mathrm{N}$ que variaram de 51,1 a 69,1 de 16,9 a $22,3 \mathrm{~kg} \cdot \mathrm{ha}^{-1} \cdot \mathrm{d}^{-1}$, valores muito superiores aos obtidos neste trabalho, porém, há de se ressaltar que o tempo de residência hidráulica da ARS foi de 18 dias, 3,75 vezes maior que o utilizado neste trabalho. Vicente-Chandler et al. (1974) observaram remoções de $338 \mathrm{~kg} \cdot \mathrm{ha}^{-1} \cdot$ ano $^{-1}$ de N, $72 \mathrm{~kg} \cdot \mathrm{ha}^{-1}$. ano ${ }^{-1}$ de P, $565 \mathrm{~kg} \cdot \mathrm{ha}^{-1} \cdot$ ano $^{-1}$ de $\mathrm{K}$, $108 \mathrm{~kg} \cdot \mathrm{ha}^{-1} \cdot \mathrm{ano}^{-1}$ de Ca e $71 \mathrm{~kg} \cdot \mathrm{ha}^{-1} \cdot \mathrm{ano}^{-1}$ de $\mathrm{Mg}$, quando cultivaram capim elefante adubado com fertilizantes inorgânicos, valores que para N, P e $\mathrm{K}$ estão inferiores aos obtidos neste trabalho. 
Quadro 2. Valores de remoção de N, P, K e Na pelo capim Napier em função das taxas de carga orgânica superficial (TCOs) aplicadas

\begin{tabular}{ccccc}
\hline \multirow{2}{*}{ TCOs $\left(\mathbf{k g . h a}^{\mathbf{- 1}} \cdot \mathbf{d}^{-\mathbf{1}}\right)$} & \multicolumn{4}{c}{ Remoção $\left(\mathbf{k g . h a} \mathbf{p}^{-\mathbf{1}}\right)$} \\
\cline { 2 - 5 } & $\mathbf{N}$ & $\mathbf{P}$ & $\mathbf{K}$ & $\mathbf{N a}$ \\
\hline 66 & 204 & 35 & 186 & 1,15 \\
130 & 248 & 38 & 156 & 0,94 \\
190 & 136 & 15 & 68 & 0,54 \\
320 & 204 & 26 & 157 & 0,83 \\
570 & 239 & 34 & 179 & 1,15 \\
\hline Média & 206 & 30 & 149 & 0,92 \\
\hline
\end{tabular}

\section{CONCLUSÕES}

Com base nos resultados obtidos, pode-se concluir que:

- o capim Napier se adaptou bem aos sistemas alagados construídos, com a aplicação da ARL, em TCOs de 66 a $570 \mathrm{~kg} \cdot \mathrm{ha}^{-1} \cdot \mathrm{d}^{-1}$, embora tenha apresentado um enraizamento predominantemente superficial no meio suporte, não apresentando problemas fisiológicos ou nutricionais em decorrência de seu cultivo nos SACs; e

- o capim Napier apresentou grande produtividade média de matéria seca e de proteína bruta e relativamente grande capacidade de remover nutrientes, especialmente nitrogênio e potássio da ARL, devendo ser considerado opção para cultivo em SACs, caso se deseje a produção de forrageiras de grande valor nutricional para alimentação animal.

\section{REFERÊNCIAS BIBLIOGRÁFICAS}

AGUIAR, E.M.; SILVA, D.A. Aspectos produtivos e morfológicos do capim elefante (Pennisetum purpureum, schum.) Cv. Roxo de Botucatu, submetido a esgoto sanitário semitratado. Encontro das Águas, 2, 1999, Montivideo. Anais..., Montevideo, 1999. p.15-18.

BRASIL, M.S. Desempenho de sistema alagado construído para tratamento de esgoto doméstico. 2005. 160f. Tese ( Doutorado em Engenharia
Agrícola), Universidade Federal de Viçosa, Viçosa.2005.

BRUNKEN, J.N. A systematic study of Pennisetum sect. Pennisetum (gramineae). American Journal of Botany, New York, 64(2): 161-176, 1977.

GOMES FILHO, R.R.G. Tratamento de Águas Residuárias da Suinocultura Utilizando o Cultivo Hidropônico de Braquiária (Brachiaria ruziziensis) e Aveia Forrageira (Avena strigosa). Viçosa: UFV, 2000. 143p. Tese de Doutorado.

GOMIDE, J.A., QUEIROZ, D.S. Valor alimentício das Brachiarias. In: Simpósio Sobre Manejo da Pastagem, 11, 1994, Piracicaba. Anais..., Piracicaba: FEALQ, 1994. p.223-248.

HUSSAR, G.J. Avaliação do desempenho de leitos cultivados no tratamento de águas residuárias de suinocultura. Campinas: FEAGRI-UNICAMP, 2001. 118p. Dissertação de Mestrado.

KIEHL, E.J. Fertilizantes orgânicos. São Paulo: Ceres, 1985, 492p.

MACHADO, R.M.G.; FREIRE, V.H.; SILVA, P.C.; FIGUERÊDO, D.V.; FERREIRA, P.E. Controle Ambiental nas Pequenas e Médias Indústrias de Laticínios. Projeto Minas Ambiente, Belo Horizonte, 2002. 224p.

PAGANINI, W.S. Disposição de esgotos no solo (Escoamento à superfície). São Paulo: Fundo editorial da AESABESP, 1997. 232p. 
SILVA, D.J. Análise de alimentos (métodos químicos e biológicos). 2. ed. Viçosa: Imprensa Universitária, UFV, 1998. 165p.

VICENTE-CHANDLER， J.V.; ALRURIA，F.;
CARO-COSTA, R.; FIGARELLO, J.; SILVA, S.; PEARSON, R.W. Intensive grassland management in the humid tropics of Puerto Rico. University of Puerto Rico, 1974. 164p. Bulletin 233. 